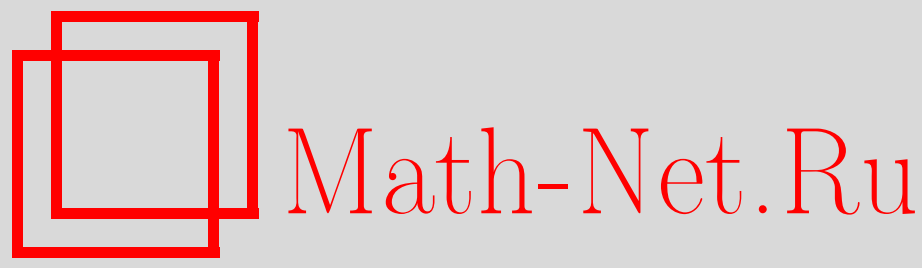

О. В. Капцов, Алгебро-геометрические структуры аналитических уравнений с частными производными, TMФ, 2016, том 189, номер 2, 219-238

DOI: https://doi.org/10.4213/tmf9089

Использование Общероссийского математического портала Math-Net.Ru подразумевает, что вы прочитали и согласны с пользовательским соглашением http://www . mathnet.ru/rus/agreement

Параметры загрузки:

IP: 18.207 .199 .55

26 апреля 2023 г., 16:08:37

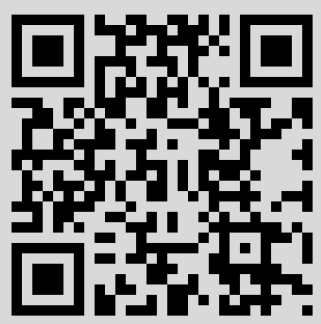


Том 189, № 2

ноябрь, 2016

\title{
$\begin{array}{ll}\text { (C) } 2016 \text { г. } & \text { O.В. Капцов }{ }^{* \dagger}\end{array}$
}

\section{АЛГЕБРО-ГЕОМЕТРИЧЕСКИЕ СТРУКТУРЫ АНАЛИТИЧЕСКИХ УРАВНЕНИЙ С ЧАСТНЫМИ ПРОИЗВОДНЫМИ}

\begin{abstract}
Изучается проблема совместности нелинейных уравнений с частными производными. Вводится алгебра сходящихся степенных рядов, модуль дифференцирований этой алгебры и модуль пфаффовых форм. Системы дифференциальных уравнений задаются степенными рядами на пространстве бесконечных джетов. Развивается техника исследования совместности дифференциальных систем, похожая на метод базисов Гребнера. Доказано, что совместные системы при некоторых предположениях порождают бесконечномерные многообразия.
\end{abstract}

Ключевые слова: совместность дифференциальных уравнений, редукция, бесконечномерное многообразие, базис Гребнера.

DOI: $10.4213 /$ tmf9089

\section{1. ВВЕДЕНИЕ}

Вопрос о совместности системы уравнений возникает одним из первых при ее исследовании. Критерий совместности линейных алгебраических уравнений дает теорема Кронекера-Капелли. В случае полиномиальных уравнений такого простого критерия нет, поэтому для исследования совместности применяется метод исключения, а также используются базисы Гребнера [1], [2]. Гораздо сложнее получить ответ для систем нелинейных уравнений с частными производными. Здесь возникают проблемы локального и глобального характера, кроме того, важно знать, каким классам гладкости принадлежат уравнения. Начиная с пионерских работ Рикье,

Работа выполнена при финансовой поддержке гранта Правительства РФ для проведения исследований под руководством ведущих ученых в Сибирском федеральном университете (договор № 14.Y26.31.0006) и Программы поддержки ведущих научных школ (гранты НШ-544.2012.1, НШ-6293.2012.9).

* Институт вычислительного моделирования СО РАН, Красноярск, Россия.

E-mail: kaptsov@icm.krasn.ru

${ }^{\dagger}$ Сибирский федеральный университет, Красноярск, Россия 
Жане [3], [4] и заканчивая современными [5]-[9], понятия, лежащие в основе совместности, - инволютивность, разрешимость - уточняются, а методы исследования меняются. В последнее время акцент исследований сместился в сторону алгоритмизации вычислений. Так, в систему компьютерной алгебры Maple [10] имплементированы алгоритмы, которые должны переводить исходную систему уравнений в некоторую "стандартную” форму. В то же время можно констатировать, что устоявшегося определения инволютивной (пассивной, стандартной) системы уравнений с частными производными нет. В настоящей работе рассматриваются пассивные системы, введенные в работах [11], [12], и изучаются их свойства.

Работа имеет следующую структуру. В разделе 2 рассматривается бесконечномерное пространство $\mathbb{K}^{T}$ отображений из счетного множества $T$ в поле $\mathbb{K}$, полное относительно некоторого нетривиального абсолютного значения. Важнейшими примерами таких полей являются поля вещественных и комплексных чисел. В пространстве $\mathbb{K}^{T}$ вводится топология прямого произведения и декартова система координат. Произвольной точке $a \in \mathbb{K}^{T}$ сопоставляется алгебра $\mathcal{F}_{a}$ сходящихся степенных рядов. Каждый ряд из $\mathcal{F}_{a}$ зависит от конечного числа переменных, однако число этих переменных может быть как угодно большим. С помощью рядов из $\mathcal{F}_{a}$ определяются аналитические функции на открытых множествах пространства $\mathbb{K}^{T}$ и аналитические отображения данного пространства. Это позволяет определить аналитические многообразия в $\mathbb{K}^{T}$. В конце раздела вводится понятие нормализованной системы образующих идеала алгебры $\mathcal{F}_{a}$. Показано, что нули аналитических функций, соответствующие нормализованной системе, задают многообразие в $\mathbb{K}^{T}$.

В разделе 3 изучаются дифференцирования алгебры $\mathcal{F}_{a}$, пфаффовы (дифференциальные) формы и производные Ли от этих форм. Доказано, что дифференцирования однозначно определяются действием на образующие алгебры $\mathcal{F}_{a}$. Вводятся инвариантные идеалы и подмодули. Далее рассматривается пространство (бесконечных) джетов $\mathbb{J}=\mathbb{K}^{T}$, где $T=\mathbb{N}_{n} \cup\left(\mathbb{N}_{m} \times \mathbb{N}^{n}\right), \mathbb{N}_{n}=1, \ldots, n$ и $\mathbb{N}$ - множество неотрицательных целых чисел. На пространстве $\mathbb{J}$ вводятся операторы полного дифференцирования, канонические формы, контактные дифференцирования и симметрии систем уравнений с частными производными. Отмечается, что задачи вычисления симметрий и законов сохранения системы уравнений тесно связаны с проблемой проверки принадлежности элемента алгебры данному дифференциальному идеалу.

В начале раздела 4 доказано, что если дифференциальные системы порождают один и тот же дифференциальный идеал, то они задают одинаковые ростки нулей. Затем определяются такие понятия, как редукция ряда относительно дифференциальной системы, условия совместности, пассивные системы. Показано, что если система $S \subset \mathcal{F}_{a}$ пассивна, то ряд $f \in \mathcal{F}_{a}$ принадлежит дифференциальному идеалу, порожденному системой $S$, тогда и только тогда, когда $f$ редуцируется к нулю относительно $S$. Основным результатом работы является теорема, в которой утверждается, что если дифференциальная система удовлетворяет некоторым условиям слабой разрешимости и совместности, то она пассивна в некоторой точке $a \in \mathbb{J}$ и задает аналитическое многообразие в окрестности этой точки. В качестве примера рассмотрено уравнение Дюбрей-Жакотен, описывающее плоские стационарные течения неоднородной жидкости. Найдено точное неинвариантное решение, зависящее от двух параметров. 


\section{2. АЛГЕБРА СХОДЯЩИХСЯ СТЕПЕННЫХ РЯДОВ И АНАЛИТИЧЕСКИЕ ФУНКЦИИ}

Пусть $\mathbb{K}$ - поле, полное относительно нетривиального абсолютного значения. Зафиксируем обозначения: $\mathbb{N}$ - множество неотрицательных целых чисел, $\mathbb{N}_{m}=$ $\{1, \ldots, m\}, \mathbb{R}_{+}-$множество положительных действительных чисел, $T$ - счетное множество, $\mathbb{K}^{T}$ - пространство отображений из $T$ в $\mathbb{K}$.

ОПРЕДЕЛЕНИЕ. Отображения $y_{t}: \mathbb{K}^{T} \rightarrow \mathbb{K}$, заданные формулой

$$
y_{t}(z)=z(t), \quad t \in T
$$

назовем декартовыми координатными функииями на $\mathbb{K}^{T}$, а значения $z(t)$ - координатами точки $z \in \mathbb{K}^{T}$.

Значение $z(t)$ удобно обозначать как $z_{t}$ по аналогии с конечномерным случаем.

Введем на пространстве $\mathbb{K}^{T}$ топологию прямого произведения, выбирая в качестве фундаментальной системы окрестностей точки $a \in \mathbb{K}^{T}$ множества

$$
U\left(a_{\tau}, \rho\right)=\left\{z \in \mathbb{K}^{T}:\left|z_{t_{i}}-a_{t_{i}}\right|<\rho_{i}, i \in \mathbb{N}_{n}\right\}
$$

здесь $t_{i} \in T, \rho_{i} \in \mathbb{R}_{+}, \rho=\left(\rho_{1}, \ldots, \rho_{n}\right), a_{\tau}=\left\{a_{t_{1}}, \ldots, a_{t_{n}}\right\}$ - множество из $n$ координат точки $a$ и $z_{t_{1}}, \ldots, z_{t_{n}}$ - координаты точки $z$. Множество (2.2) будем называть брусом.

Пусть $\left\{X_{t}\right\}_{t \in T}-$ множество символов,

$$
\tau=\left\{t_{1}, \ldots, t_{n}\right\} \subset T, \quad \rho=\left(\rho_{1}, \ldots, \rho_{n}\right) \in \mathbb{R}_{+}^{n}, \quad a_{\tau}=\left\{a_{t_{1}}, \ldots, a_{t_{1}}\right\} \subset \mathbb{K} .
$$

Обозначим через $A\left(a_{\tau}, \rho\right)$ множество степенных рядов вида

$$
f=\sum_{\alpha \in \mathbb{N}^{n}} c_{\alpha}\left(X_{t_{1}}-a_{t_{1}}\right)^{\alpha_{1}} \ldots\left(X_{t_{n}}-a_{t_{n}}\right)^{\alpha_{n}},
$$

где $c_{\alpha} \in \mathbb{K}, \alpha=\left(\alpha_{1}, \ldots, \alpha_{n}\right) \in \mathbb{N}^{n}$, для которых конечна величина

$$
\|f\|_{\rho}=\sum_{\alpha \in \mathbb{N}^{n}}\left|c_{\alpha}\right| \rho_{1}^{\alpha_{1}} \ldots \rho_{n}^{\alpha_{n}} .
$$

Как следует из книги [13], $A\left(a_{\tau}, \rho\right)$ - банахова алгебра с нормой $(2.4)$.

Введем отношение $\prec$ на $\mathbb{R}_{+}^{n}$. Пусть $\rho=\left(\rho_{1}, \ldots, \rho_{n}\right), \rho^{*}=\left(\rho_{1}^{*}, \ldots, \rho_{n}^{*}\right)$; будем считать $\rho \prec \rho^{*}$, если разность $\rho_{i}^{*}-\rho_{i}$ положительна для всех $i \in \mathbb{N}_{n}$.

УТВЕРЖДЕНИЕ 2.1. Пусть

$$
\begin{gathered}
\tau=\left\{t_{1}, \ldots, t_{n}\right\} \subset \tau^{\prime}=\left\{t_{1}, \ldots, t_{m}\right\} \subset T, \quad n<m, \\
\rho=\left(\rho_{1}, \ldots, \rho_{n}\right) \prec \rho^{*}=\left(\rho_{1}^{*}, \ldots, \rho_{n}^{*}\right), \quad \rho^{\prime}=\left(\rho_{1}, \ldots, \rho_{n}, 1, \ldots, 1\right) \in \mathbb{R}_{+}^{m} .
\end{gathered}
$$

Тогда алгебра $A\left(a_{\tau}, \rho^{*}\right)$ вкладъвается в алгебру $A\left(a_{\tau}, \rho\right)$, а алгебра $A\left(a_{\tau}, \rho\right)$ вкладъвается в алгебру $A\left(a_{\tau^{\prime}}, \rho^{\prime}\right)$ и для всех $i \in \mathbb{N}_{n}$ справедливы включения

$$
\frac{\partial}{\partial X_{t_{i}}} A\left(a_{\tau}, \rho^{*}\right) \subset A\left(a_{\tau}, \rho\right) .
$$


ДокАЗАтЕЛЬСтво. Вложения очевидны, а формула (2.5) следует из аналогичной формулы в книге [13].

ОПРЕДЕЛЕНИЕ. Степенной ряд $f$ вида (2.3) называется сходящимся (в окрестности точки $\left.a \in \mathbb{K}^{T}\right)$, если $f \in A\left(a_{\tau}, \rho\right)$ для некоторого $\rho \in \mathbb{R}_{+}^{n}$ и $a_{\tau}=\left\{a_{t_{1}}, \ldots, a_{t_{n}}\right\} \subset \mathbb{K}$, где $a_{t_{1}}, \ldots, a_{t_{n}}-$ координаты точки $a \in \mathbb{K}^{T}$.

Для каждой точки $a \in \mathbb{K}^{T}$ с частью координат $a_{\tau}=\left\{a_{t_{1}}, \ldots, a_{t_{n}}\right\}$ рассмотрим объединение (не дизъюнктное) алгебр

$$
\mathcal{F}_{a}=\bigcup_{\substack{\rho \in \mathbb{R}_{+}^{n}, n \in N_{0}, a_{\tau} \subset \mathbb{K}}} A\left(a_{\tau}, \rho\right),
$$

где $N_{0}=\mathbb{N} \backslash\{0\}$. Множество $\mathcal{F}_{a}$ является $\mathbb{K}$-алгеброй сходящихся степенных рядов.

С помощью сходящихся степенных рядов из $\mathcal{F}_{a}$ введем аналитические функции на открытых множествах пространства $\mathbb{K}^{T}$. Каждый ряд $f \in A\left(a_{\tau}, \rho\right)$ вида (2.3) порождает функцию $\tilde{f}$ следующим образом. Пусть $\tau=\left\{t_{1}, \ldots, t_{n}\right\} \subset T$, $\rho=\left(\rho_{1}, \ldots, \rho_{n}\right) \in \mathbb{R}_{+}^{n}$ и $a_{\tau}=\left\{a_{t_{1}}, \ldots, a_{t_{n}}\right\} \subset \mathbb{K}$. Согласно определению алгебры $A\left(a_{\tau}, \rho\right)$ ряд $f$ сходится в полицилиндре

$$
\Pi\left(a_{\tau}, \rho\right)=\left\{\left(z_{t_{1}}, \ldots, z_{t_{n}}\right) \in \mathbb{K}^{n}:\left|z_{t_{i}}-a_{t_{i}}\right|<\rho_{i}, i \in \mathbb{N}_{n}\right\} .
$$

Этому полицилиндру однозначно соответствует брус $U\left(a_{\tau}, \rho\right)$ вида $(2.2)$. Тогда для любой точки $z \in U\left(a_{\tau}, \rho\right)$ функция $\tilde{f}$ задается формулой

$$
\tilde{f}(z)=f\left(z_{\tau}\right)=\sum_{\alpha \in \mathbb{N}^{n}} c_{\alpha}\left(z_{t_{1}}-a_{t_{1}}\right)^{\alpha_{1}} \ldots\left(z_{t_{n}}-a_{t_{n}}\right)^{\alpha_{n}},
$$

где $z_{\tau}=\left(z_{t_{1}}, \ldots, z_{t_{n}}\right) \in \Pi\left(a_{\tau}, \rho\right)$. Таким образом, функция $\tilde{f}$ зависит от конечного числа переменных. Построенную функцию $\tilde{f}$ будем называть локально-аналитической. Это позволяет по аналогии с конечномерным случаем [14] ввести аналитические функции в открытых множествах.

ОПредЕлЕниЕ. Пусть $U$ - открытое множество в $\mathbb{K}^{T}$. Функция $h: U \rightarrow \mathbb{K}$ называется аналитической в $U$, если для каждой точки $z \in U$ существует брус $U\left(a_{\tau}, \rho\right)$ и локально-аналитическая функция $\tilde{f}$ в этом брусе такая, что $h(z)=\tilde{f}(z)$ для всех $z \in U\left(a_{\tau}, \rho\right)$.

ЗАмЕЧАНИЕ. Всюду в дальнейшем для $E \subset \mathcal{F}_{a}$

$$
\widetilde{E}=\{\tilde{f}: f \in E\}
$$

обозначает множество локально-аналитических функций $\tilde{f}$, соответствующих рядам $f \in \mathcal{F}_{a}$. Можно показать, следуя монографии [15], что функция, локальноаналитическая в брусе $U\left(a_{\tau}, \rho\right)$, является аналитической в $U\left(a_{\tau}, \rho\right)$.

ОПрЕДЕЛЕНИЕ. Пусть $U$ - открытое множество в $\mathbb{K}^{T}$. Отображение $\phi: U \rightarrow \mathbb{K}^{T}$ с компонентами $\phi_{t}, t \in T$, называется аналитическим в $U$, если каждая функция $\phi_{t}$ является аналитической в $U$. Отображение $\phi$ называется бианалитическим, если у него существует обратное аналитическое отображение $\phi^{-1}$. 
ОПРЕДЕЛЕНИЕ. Множество

$$
C_{S}=\left\{z \in \mathbb{K}^{T}: z(t)=0 \text { для всех } t \in S \subset T\right\}
$$

называется координатным подпространством в $\mathbb{K}^{T}$.

Нижеследующее определение многообразия является прямым обобщением конечномерной конструкции.

ОПрЕДЕлЕНиЕ. Множество $M \subset \mathbb{K}^{T}$ называется многообразием в $\mathbb{K}^{T}$, если для любой точки $z \in M$ существуют открытые множества $U, U^{\prime}$ в $\mathbb{K}^{T}$, причем $z \in U$, и бианалитическое отображение $\phi: U \rightarrow U^{\prime}$ такое, что

$$
\phi(U \cap M)=U^{\prime} \cap C_{S},
$$

где $C_{S}$ - некоторое координатное подпространство в $\mathbb{K}^{T}$. При этом ограничение $\bar{\phi}=\left.\phi\right|_{M \cap U}$ отображения $\phi$ на $U \cap M$ называется локальной системой координат на $U \cap M$, а множество переменных (декартовых координатных функций на $C_{S}$ ), от которых зависит обратное отображение $\bar{\phi}^{-1}$, называется набором параметров многообразия.

Аналитические функции и многообразия в координатных подпространствах вводятся аналогичным образом.

Обозначим через $\operatorname{iv}(f)$ множество символов, от которых зависит ряд $f \in \mathcal{F}_{a}$. Если $E \subset \mathcal{F}_{a}$, то

$$
\operatorname{iv}(E)=\{\operatorname{iv}(f): f \in E\} .
$$

ОПредЕлЕНиЕ. Пусть $\mathcal{R}$ - подалгебра алгебры $\mathcal{F}_{a}$ и $I-$ идеал в $\mathcal{R}$. Система образующих $\mathcal{B}$ идеала $I$ называется нормализованной, если:

1) любой элемент $f \in \mathcal{B}$ имеет вид $f=X_{s}+g$, причем элементы $X_{s}$ образуют подмножество $L \subset\left\{X_{t}\right\}_{t \in T}$;

2) если $f_{1}=X_{t}+g_{1} \in \mathcal{B}$ и $f_{2}=X_{t}+g_{2} \in \mathcal{B}$, то $g_{1}=g_{2}$.

В этом случае $L$ называется множеством главных переменных системы $\mathcal{B}$.

Предположим, что $T^{\prime} \subset T$. Тогда множество

$$
C\left(T^{\prime}\right)=\left\{z \in \mathbb{K}^{T}: z(t)=0 \text { для всех } t \in T \backslash T^{\prime}\right\}
$$

является координатным подпространством в $\mathbb{K}^{T}$. Топология в $C\left(T^{\prime}\right)$ индуцирована топологией в $\mathbb{K}^{T}$. Аналитические функции в открытых множествах подпространства $C\left(T^{\prime}\right)$ определяются с помощью степенных рядов и формулы (2.7). Множество рядов

$$
\mathcal{F}_{a}\left(T^{\prime}\right)=\left\{f \in \mathcal{F}_{a}: \operatorname{iv}(f) \subseteq\left\{X_{t}\right\}_{t \in T^{\prime}}\right\}
$$

образует подалгебру алгебры $\mathcal{F}_{a}$.

УтВЕРЖДЕНИЕ 2.2. Пусть $T^{\prime} \subseteq T, \mathcal{B}$ - нормализованная система образующих идеала подалгебры $\mathcal{F}_{a}\left(T^{\prime}\right)(2.11)$ и $\widetilde{\mathcal{B}}$ - соответствующее множество аналитических функиий в некотором открытом множестве $V$ координатного подпространства (2.10), $L$-множество главных переменных системы $\mathcal{B}, S=\left\{s \in T^{\prime}: X_{s} \in L\right\}$, $T^{\prime \prime}=T^{\prime} \backslash S$. Тогда множество

$$
Z(\widetilde{\mathcal{B}})=\{z \in V: \tilde{f}(z)=0 \text { для всех } f \in \widetilde{\mathcal{B}}\}
$$


является многообразием в подпространстве $C\left(T^{\prime}\right)$ и множество декартовьх координатных функиий $\left\{y_{t}\right\}_{t \in T^{\prime \prime}}$ образует набор параметров многообразия.

ДокАзАтЕльство. Отображение $\phi$ задается формулами $y_{t}^{\prime}=y_{t}+g_{t}, y_{s}^{\prime}=y_{s}$, где $y_{t}$ соответствует символу $X_{t} \in L$ и $\mathrm{iv}\left(g_{t}\right), y_{s} \in\left\{y_{q}\right\}_{q \in T^{\prime \prime}}$. Следовательно, обратное отображение $\phi^{-1}$ имеет вид $y_{t}=y_{t}^{\prime}-g_{t}, y_{s}=y_{s}^{\prime}$. Очевидно, ограничение отображения $\phi$ на $Z(\widetilde{\mathcal{B}})$ является проекцией $Z(\widetilde{\mathcal{B}})$ на $V \cap C\left(T^{\prime \prime}\right)$. Таким образом, множество $Z(\widetilde{\mathcal{B}})$ - многообразие.

ЗАмЕчАниЕ. Важно отметить, что в утверждении 2.2 требуется, чтобы все функции из $\widetilde{\mathcal{B}}$ были заданы на одном и том же открытом множестве $V$.

\section{3. ДИФФЕРЕНЦИРОВАНИЯ И ЛОКАЛЬНЫЕ СИСТЕМЫ}

Напомним, что дифференцированием коммутативной алгебры $A$ над полем $K$ называется $K$-линейное отображение $\mathcal{D}: A \rightarrow A$, для которого $\mathcal{D}(a b)=a \mathcal{D}(b)+b \mathcal{D}(a)$.

Лемма 3.1. Произвольное дифференцирование $\mathcal{D}$ алгебры $\mathcal{F}_{a}$ однозначно определяется значениями на координатных функииях $y_{t}$ и задается для любого $f \in \mathcal{F}_{a}$ формулой

$$
\mathcal{D}(f)=\sum_{t \in T} \mathcal{D}\left(y_{t}\right) \frac{\partial f}{\partial y_{t}} .
$$

ДокАЗАТЕЛЬство. Не ограничивая общности, можно считать, что $a=0 \in \mathbb{K}^{T}$. Моном $y_{t_{1}}^{\alpha_{1}} \ldots y_{t_{n}}^{\alpha_{n}}$ обозначим через $y^{\alpha}$. Пусть полином $p=\sum_{\alpha \in A} c_{\alpha} y^{\alpha}$, где $c_{\alpha} \in \mathbb{K}$, $A$ - конечное подмножество в $\mathbb{N}^{n}$. Согласно определению дифференцирования верны формулы

$$
\begin{aligned}
\mathcal{D}\left(y^{\alpha}\right) & =\sum_{i=1}^{n} \alpha_{i} \mathcal{D}\left(y_{t_{i}}\right) y_{t_{1}}^{\alpha_{1}} \ldots y_{t_{i}}^{\alpha_{i}-1} \ldots y_{t_{n}}^{\alpha_{n}}=\sum_{i=1}^{n} \mathcal{D}\left(y_{t_{i}}\right) \frac{\partial y^{\alpha}}{\partial y_{t_{i}}} \\
\mathcal{D}(p) & =\sum_{\alpha \in A} c_{\alpha} \mathcal{D}\left(y^{\alpha}\right)=\sum_{\alpha \in A} c_{\alpha} \sum_{i=1}^{n} \mathcal{D}\left(y_{t_{i}}\right) \frac{\partial y^{\alpha}}{\partial y_{t_{i}}}=\sum_{i=1}^{n} \mathcal{D}\left(y_{t_{i}}\right) \frac{\partial p}{\partial y_{t_{i}}} .
\end{aligned}
$$

Докажем, что дифференцирование $\mathcal{D}$ однозначно продолжается с алгебры полиномов на алгебру $\mathcal{F}_{0}$. Предположим, что существует другое дифференцирование $\mathcal{D}_{0}$ алгебры $\mathcal{F}_{0}$, совпадающее с $\mathcal{D}$ на полиномах. Тогда дифференцирование $\mathcal{D}^{*}=\mathcal{D}-\mathcal{D}_{0}$ обращает в ноль любой многочлен.

Предположим, что существует ряд $f \in \mathcal{F}_{0}$ такой, что $\mathcal{D}^{*}(f) \neq 0$. Напомним [16], что порядком ряда $f \neq 0$ (обозначается $\operatorname{ord}(f))$ называется наименьшее натуральное число $q$ такое, что однородная часть степени $q$ ряда $f$ не равна нулю.

Для любого полинома $p \in \mathcal{F}_{0}$ верно равенство $\mathcal{D}^{*}(f)=\mathcal{D}^{*}(f-p)$, следовательно,

$$
\operatorname{ord}\left(\mathcal{D}^{*}(f)\right)=\operatorname{ord}\left(\mathcal{D}^{*}(f-p)\right) \text {. }
$$

Кроме того, в силу формул (3.1) верно неравенство $\operatorname{ord}\left(\mathcal{D}^{*}(f)\right) \geqslant \operatorname{ord}(f)-1$. Поэтому, выбирая полином $p$, "уничтожающий младшие члены" ряда $f$, можно сделать $\operatorname{ord}\left(\mathcal{D}^{*}(f-p)\right)$ сколь угодно большим числом. Но это противоречит (3.2), так как порядок ряда $\mathcal{D}^{*}(f)$ - конечное число. Лемма доказана. 
Очевидно, множество дифференцирований алгебры $\mathcal{F}_{a}$ образует модуль над $\mathcal{F}_{a}$. Обозначим его как $\operatorname{Der}_{a}$. Хорошо известно [16], что множество дифференцирований любой коммутативной алгебры над полем образует алгебру Ли с коммутатором

$$
\left[\mathcal{D}_{1}, \mathcal{D}_{2}\right]=\mathcal{D}_{1} \mathcal{D}_{2}-\mathcal{D}_{2} \mathcal{D}_{1}
$$

Следовательно, Der ${ }_{a}$ - алгебра Ли над полем $\mathbb{K}$. В силу леммы 3.1 дифференцирования алгебры $\mathcal{F}_{a}$ можно также называть локальными векторными полями в окрестности точки $a \in \mathbb{K}^{T}$.

Следуя монографии [16], назовем дифференциалом ряда $f \in \mathcal{F}_{a}$ линейное отображение $d f: \operatorname{Der}_{a} \rightarrow \mathcal{F}_{a}$, действующее по формуле

$$
d f(\mathcal{D})=\mathcal{D}(f)
$$

Очевидно, множество дифференциалов рядов из $f \in \mathcal{F}_{a}$ порождает модуль над $\mathcal{F}_{a}$. Этот модуль обозначается как $\operatorname{Der}_{a}^{*}$ и называется модулем пфаффовых форм. Элементами данного модуля являются конечные суммы вида $\sum g_{t} d f_{t}$, где $g_{t}, f_{t} \in \mathcal{F}_{a}$.

УтвЕРЖДЕНИЕ 3.1. Модуль пфаффовых форм Der* порожден дифференииалами декартовых координатных функиий, т.е. элементами $\left\{d y_{t}\right\}_{t \in T}$.

ДокАзАтЕльство. Достаточно показать, что дифференциал $d f$ задается классической формулой

$$
d f=\sum_{t \in T} \frac{\partial f}{\partial y_{t}} d y_{t}
$$

здесь суммирование в правой части конечное, так как $f$ зависит от конечного числа переменных. Возьмем произвольное дифференцирование $\mathcal{D} \in \operatorname{Der}_{a}$ и сравним значения левой и правой части равенства (3.3) на $\mathcal{D}$ :

$$
d f(\mathcal{D})=\mathcal{D}(f)=\sum_{t} \mathcal{D}\left(y_{t}\right) \frac{\partial f}{\partial y_{t}}, \quad \sum_{t} \frac{\partial f}{\partial y_{t}} d y_{t}(\mathcal{D})=\sum_{t} \frac{\partial f}{\partial y_{t}} \mathcal{D}\left(y_{t}\right) .
$$

Мы видим, что эти значения совпадают.

ОПРЕДЕЛЕНИЕ. Производной Ли, порожденной дифференцированием $\mathcal{D} \in \operatorname{Der}_{a}$, называется $\mathbb{K}$-линейное отображение $\mathcal{L}_{\mathcal{D}}: \operatorname{Der}_{a}^{*} \rightarrow \operatorname{Der}_{a}^{*}$, удовлетворяющее условию

$$
\mathcal{L}_{\mathcal{D}}(g d f)=\mathcal{D}(g) d f+g d \mathcal{D}(f), \quad f, g \in \mathcal{F}_{a} .
$$

УтВЕРЖДЕНИЕ 3.2. Множество производных Ли образует алгебру Ли над полем $\mathbb{K}$, и для всех $k_{1}, k_{2} \in \mathbb{K} u$ любъх $\mathcal{D}_{1}, \mathcal{D}_{2} \in \operatorname{Der}_{a}$ справедливы равенства

$$
\begin{gathered}
k_{1} \mathcal{L}_{\mathcal{D}_{1}}+k_{2} \mathcal{L}_{\mathcal{D}_{2}}=\mathcal{L}_{k_{1} \mathcal{D}_{1}+k_{2} \mathcal{D}_{2}}, \\
{\left[\mathcal{L}_{\mathcal{D}_{1}}, \mathcal{L}_{\mathcal{D}_{2}}\right]=\mathcal{L}_{\left[\mathcal{D}_{1}, \mathcal{D}_{2}\right]} .}
\end{gathered}
$$

ДокАзАТЕльство. Для доказательства утверждения достаточно проверить формулы (3.5), (3.6) на произвольной пфаффовой форме $\omega=\sum_{t \in \tau} f_{t} d y_{t}$, где $\tau$ - конечное подмножество в $T$. Равенство левой и правой части формулы (3.5) элементарно проверяется действием на форму $\omega$. 
Покажем, что

$$
\mathcal{L}_{\mathcal{D}_{1}} \mathcal{L}_{\mathcal{D}_{2}}(\omega)-\mathcal{L}_{\mathcal{D}_{2}} \mathcal{L}_{\mathcal{D}_{1}}(\omega)=\mathcal{L}_{\left[\mathcal{D}_{1}, \mathcal{D}_{2}\right]}(\omega)
$$

Для этого вычислим $\mathcal{L}_{\mathcal{D}_{1}} \mathcal{L}_{\mathcal{D}_{2}}(\omega)$ и $\mathcal{L}_{\mathcal{D}_{2}} \mathcal{L}_{\mathcal{D}_{1}}(\omega)$ : имеем

$$
\begin{aligned}
\mathcal{L}_{\mathcal{D}_{1}} \mathcal{L}_{\mathcal{D}_{2}}(\omega)= & \mathcal{L}_{\mathcal{D}_{1}} \mathcal{L}_{\mathcal{D}_{2}}\left(\sum_{t} f_{t} d y_{t}\right)=\mathcal{L}_{\mathcal{D}_{1}}\left(\sum_{t} \mathcal{D}_{2}\left(f_{t}\right) d y_{t}+\sum_{t} f_{t} d \mathcal{D}_{2}\left(y_{t}\right)\right)= \\
=\sum_{t} \mathcal{D}_{1} \mathcal{D}_{2}\left(f_{t}\right) d y_{t} & +\sum_{t} \mathcal{D}_{2}\left(f_{t}\right) d \mathcal{D}_{1}\left(y_{t}\right)+ \\
& +\sum_{t} \mathcal{D}_{1}\left(f_{t}\right) d \mathcal{D}_{2}\left(y_{t}\right)+\sum_{t} f_{t} d \mathcal{D}_{1} \mathcal{D}_{2}\left(y_{t}\right), \\
\mathcal{L}_{\mathcal{D}_{2}} \mathcal{L}_{\mathcal{D}_{1}}(\omega)=\sum_{t} \mathcal{D}_{2} \mathcal{D}_{1}\left(f_{t}\right) d y_{t} & +\sum_{t} \mathcal{D}_{1}\left(f_{t}\right) d \mathcal{D}_{2}\left(y_{t}\right)+ \\
& +\sum_{t} \mathcal{D}_{2}\left(f_{t}\right) d \mathcal{D}_{1}\left(y_{t}\right)+\sum_{t} f_{t} d \mathcal{D}_{2} \mathcal{D}_{1}\left(y_{t}\right) .
\end{aligned}
$$

Следовательно, верны равенства

$$
\begin{aligned}
\left(\mathcal{L}_{\mathcal{D}_{1}} \mathcal{L}_{\mathcal{D}_{2}}\right. & \left.-\mathcal{L}_{\mathcal{D}_{2}} \mathcal{L}_{\mathcal{D}_{1}}\right)(\omega)= \\
& =\sum_{t}\left(\mathcal{D}_{1} \mathcal{D}_{2}\left(f_{t}\right)-\mathcal{D}_{2} \mathcal{D}_{1}\left(f_{t}\right)\right) d y_{t}+\sum_{t} f_{t} d\left(\mathcal{D}_{1} \mathcal{D}_{2}\left(y_{t}\right)-\mathcal{D}_{2} \mathcal{D}_{1}\left(y_{t}\right)\right)= \\
& =\sum_{t}\left[\mathcal{D}_{1}, \mathcal{D}_{2}\right]\left(f_{t}\right) d y_{t}+\sum_{t} f_{t} d\left(\left[\mathcal{D}_{1}, \mathcal{D}_{2}\right]\left(y_{t}\right)\right)=\mathcal{L}_{\left[\mathcal{D}_{1}, \mathcal{D}_{2}\right]}(\omega) .
\end{aligned}
$$

ОПРЕДЕЛЕНИЕ. Идеал $I$ алгебры $\mathcal{F}_{a}$ называется инвариантным относительно дифференцирования $\mathcal{D} \in \operatorname{Der}_{a}$, если $\mathcal{D}(I) \subset I$. Подмодуль $\mathcal{M}$ модуля пфаффовых форм Der* ${ }_{a}^{*}$ называется инвариантным относительно дифференцирования $\mathcal{D} \in \operatorname{Der}_{a}$, если $\mathcal{L}_{\mathcal{D}}(\mathcal{M}) \subset \mathcal{M}$.

УТВЕРЖДЕНИЕ 3.3. Множество дифферениирований, оставляющих инвариантным идеал $I \subset \mathcal{F}_{a}$ или подмодуль $\mathcal{M} \subset \operatorname{Der}_{a}^{*}$, образует алгебру Ли над полем $\mathbb{K}$.

ДоказАтеЛьство. Пусть $\mathcal{D}_{1}, \mathcal{D}_{2} \in \operatorname{Der}_{a}$ и $\mathcal{D}_{i}(I) \subset I$. Тогда имеем $\mathcal{D}_{1} \mathcal{D}_{2}(I) \subset I$ и $\mathcal{D}_{2} \mathcal{D}_{1}(I) \subset I$. Следовательно, $\left[\mathcal{D}_{1}, \mathcal{D}_{2}\right] \subset I$. Аналогичные рассуждения проводятся для подмодуля $\mathcal{M}$.

Всюду в дальнейшем мы полагаем $T=\mathbb{N}_{n} \cup\left(\mathbb{N}_{m} \times \mathbb{N}^{n}\right)$. В этом случае пространство $\mathbb{K}^{T}$ называется пространством джетов и обозначается через $\mathbb{J}$. Декартовы координатные функции на $\mathbb{J}$ обозначаются через $x_{i}, u_{\alpha}^{j}$, где $i \in \mathbb{N}_{n}, j \in \mathbb{N}_{m}, \alpha \in \mathbb{N}^{n}$. Множество $Y$ декартовых координатных функций разбивается на два подмножества

$$
X=\left\{x_{1}, \ldots, x_{n}\right\}, \quad U=\left\{u_{\alpha}^{j}\right\}_{\alpha \in \mathbb{N}^{n}}^{j \in \mathbb{N}_{m}}
$$

Введем $n$ отображений $D_{1}, \ldots, D_{n}$ из $Y$ в $Y$ :

$$
D_{k}\left(x_{k}\right)=1, \quad D_{k}\left(x_{i}\right)=0 \quad \text { при } \quad i \neq k, \quad D_{k}\left(u_{\alpha}^{j}\right)=u_{\alpha+e_{k}}^{j},
$$


где $k \in \mathbb{N}_{n}, e_{1}=(1,0, \ldots, 0), \ldots, e_{n}=(0, \ldots, 0,1)$ - элементы из $\mathbb{N}^{n}$. Тогда согласно лемме 3.1 эти отображения единственным образом продолжаются до дифференцирования алгебры $\mathcal{F}_{a}$ и при произвольном $f \in \mathcal{F}_{a}$ задаются формулами

$$
D_{k}(f)=\frac{\partial f}{\partial x_{k}}+\sum_{\substack{j \in \mathbb{N}_{m} \\ \alpha \in \mathbb{N}^{n}}} \frac{\partial f}{\partial u_{\alpha}^{j}} u_{\alpha+e_{k}}^{j} .
$$

Дифференцирования $D_{k}$ часто называют операторами полного дифферениирования [17], так как формула (3.8) представляет собой цепное правило.

Таким образом, алгебра $\mathcal{F}_{a}$ сходящихся степенных рядов с $n$ операторами полного дифференцирования является дифференциальной алгеброй. Произведение операторов $D_{1}^{\alpha_{1}} \cdots D_{n}^{\alpha_{n}}$ будем обозначать как $D^{\alpha}$, где $\alpha=\left(\alpha_{1}, \ldots, \alpha_{n}\right)$. Дифференциальный идеал алгебры $\mathcal{F}_{a}$, порожденный множеством $S \subset \mathcal{F}_{a}$, будем обозначать как $\langle\langle S\rangle$.

Напомним, что локальной аналитической системой уравнений с частными производными в работе [11] называлась тройка множеств $(\mathbb{K}, Y, S)$, где $S$ - конечное подмножество в $\mathcal{F}_{a}$. В дальнейшем для краткости конечное множество $S \subset \mathcal{F}_{a}$ мы будем называть локальной дифференциальной системой.

Рассмотрим счетное множество $\Omega=\left\{\omega_{\alpha}^{i}\right\}_{\alpha \in \mathbb{N}^{n}}^{i \in \mathbb{N}_{m}}$ канонических пфаффовых форм

$$
\omega_{\alpha}^{i}=d u_{\alpha}^{i}-\sum_{j=1}^{n} u_{\alpha+e_{j}}^{i} d x_{j} .
$$

Обозначим через $\mathcal{P}_{a}$ подмодуль модуля $\operatorname{Der}_{a}^{*}$, порожденный этими каноническими формами. Напомним, что $\mathcal{L}_{\mathcal{D}}$ обозначает производную Ли, порожденную дифференцированием $\mathcal{D}$.

ОПРЕДЕЛЕНИЕ. Дифференцирование $\mathcal{D} \in \operatorname{Der}_{a}$ называется контактным, если выполнено условие $\mathcal{L}_{\mathcal{D}}\left(\mathcal{P}_{a}\right) \subseteq \mathcal{P}_{a}$.

Очевидно, что операторы полного дифференцирования $D_{1}, \ldots, D_{n}(3.8)$ являются контактными дифференцированиями. Если $\mathcal{D}$ - контактное дифференцирование, то операторы

$$
\mathcal{D}-\sum_{j=1}^{n} \mathcal{D}\left(x_{j}\right) D_{j}
$$

также являются контактными дифференцированиями. Поэтому, не ограничивая общности, можно рассматривать контактные дифференцирования вида

$$
\mathcal{D}=\sum_{\substack{i \in \mathbb{N}_{m} \\ \alpha \in \mathbb{N}^{n}}} \mathcal{D}\left(u_{\alpha}^{i}\right) \frac{\partial}{\partial u_{\alpha}^{i}} .
$$

Такие дифференцирования часто называют вертикальными [18].

Фактически повторяя известные рассуждения [17], [18], можно показать, что дифференцирование $(3.9)$ является контактным тогда и только тогда, когда $\left[\mathcal{D}, D_{i}\right]=0$ для всех $i \in \mathbb{N}_{n}$. Это означает, что коэффициенты $\mathcal{D}\left(u_{\alpha}^{i}\right)$ в $(3.9)$ задаются формулой $\mathcal{D}\left(u_{\alpha}^{i}\right)=D^{\alpha} \mathcal{D}\left(u^{i}\right)$. 
ОПРЕДЕЛЕНИЕ. Контактное дифференцирование $\mathcal{D} \in \operatorname{Der}_{a}$ называется (инфинитезимальной) симметрией локальной дифференциальной системы

$$
S=\left\{f_{1}, \ldots, f_{k}\right\} \subset \mathcal{F}_{a},
$$

если дифференциальный идеал $I=\langle\langle S\rangle\rangle$ инвариантен относительно $\mathcal{D}$, т. е. $\mathcal{D}(I) \subseteq I$.

Следует отметить, что инвариантность идеала $\langle\langle S\rangle\rangle$ достаточно проверить на любой дифференциальной системе образующих. Точнее, справедливо

УтвеРЖДЕНИЕ 3.4. Пусть $S$ - локальная дифберенииальная система и $\mathcal{D}$ - контактное дифференцирование такое, что $\mathcal{D}(S) \subseteq\langle\langle S\rangle$. Тогда $\mathcal{D}$ - симметрия системы $S$.

ДокАЗАтЕЛЬСтво. Пусть $f \in\langle\langle S\rangle\rangle$, тогда

$$
f=\sum_{\substack{i \in \mathbb{N}_{k}, \alpha \in A}} a_{\alpha}^{i} D^{\alpha} f_{i},
$$

где $a_{\alpha}^{i} \in \mathcal{F}_{a}, A-$ конечное подмножество в $\mathbb{N}^{n}, f_{i} \in S$. Требуется доказать, что $\mathcal{D}(f) \in\langle\langle S\rangle$. По определению дифференцирования имеем

$$
\mathcal{D}(f)=\sum \mathcal{D}\left(a_{\alpha}^{i}\right) D^{\alpha} f_{i}+\sum a_{\alpha}^{i} \mathcal{D} D^{\alpha} f_{i}
$$

Не ограничивая общности, можно считать дифференцирование вертикальным, таким образом, последнее равенство переписывается в виде

$$
\mathcal{D}(f)=\sum \mathcal{D}\left(a_{\alpha}^{i}\right) D^{\alpha} f_{i}+\sum a_{\alpha}^{i} D^{\alpha} \mathcal{D}\left(f_{i}\right)
$$

Каждое слагаемое в правой части последнего равенства лежит в идеале $\langle\langle S\rangle$. Следовательно, левая часть равенства принадлежит идеалу.

ЗАмЕЧАниЕ. Приведенное выше определение симметрии, сформулированное с других позиций, можно найти в коллективной монографии [18].

Понятия, в которых используются дифференциальные идеалы, применимы к законам сохранения и различным определяющим уравнениям [19]. Так, закон сохранения можно ввести следующим образом.

ОПРЕДЕЛЕНИЕ. Законом сохранения дифференциальной системы $S \subset \mathcal{F}_{a}$ будем называть кортеж $\left(g_{1}, \ldots, g_{n}\right) \in \mathcal{F}_{a}^{n}$ такой, что

$$
D_{1} g_{1}+\cdots+D_{n} g_{n} \in\langle\langle S\rangle\rangle
$$

Возникает вопрос о проверке принадлежности элемента идеалу. В алгебре многочленов для этого полезно использовать базис Гребнера идеала [1]. Аналогом базиса Гребнера в нашем случае является пассивная система. Это понятие и его применения рассматриваются в следующем разделе. 


\section{4. МНОГООБРАЗИЯ, ПОРОЖДДЕННЫЕ СИСТЕМАМИ}

В этом разделе мы доказываем основные результаты работы, но сначала, используя обозначение (2.8), сформулируем утверждение, которое оказывается весьма полезным в дальнейшем.

УтвеРжДение 4.1. Пусть $S=\left\{f_{1}, \ldots, f_{k}\right\} \subset \mathcal{F}_{a}$ - локальнал дифференииальная система. Тогда существует брус $U\left(a_{\tau}, \rho\right)$ вида $(2.2)$ такой, что функиии $\widetilde{D^{\alpha} f_{i}}$ являются аналитическими в этом брусе для всех $i \in \mathbb{N}_{k}$ и любых $\alpha \in \mathbb{N}^{n}$.

ДокАзАТЕЛЬСтво. Очевидно, существует полицилинр П $\left(a_{\tau}, \rho^{*}\right)$ вида $(2.6)$, в котором сходятся ряды $f_{1}, \ldots, f_{k}$, т. е. $S \subset A\left(a_{\tau}, \rho^{*}\right)$. Согласно формуле дифференцирования (3.8) имеем

$$
D_{i}\left(f_{s}\right)=\frac{\partial f_{s}}{\partial x_{i}}+\sum_{\substack{j \in \mathbb{N}_{m} \\ \alpha \in \mathbb{N}^{n}}} \frac{\partial f_{s}}{\partial u_{\alpha}^{j}} u_{\alpha+e_{i}}^{j}, \quad s \in \mathbb{N}_{k},
$$

причем сумма в правой части состоит из конечного числа слагаемых. Кроме того, в силу утверждения 2.1 частные производные $\partial f_{s} / \partial x_{i}$ и $\partial f_{s} / \partial u^{j}$ принадлежат алгебpe $A\left(a_{\tau}, \rho\right)$ при любом $\rho \prec \rho^{*}$. Таким образом, соответствующие функции $\widetilde{\partial f_{s} / \partial x_{i}}$ и $\partial \widetilde{f_{s} / \partial u^{j}}$ являются аналитическими в брусе $U\left(a_{\tau}, \rho\right)$. Поскольку $\rho-$ произвольный кортеж, удовлетворяющий условию $\rho \prec \rho^{*}$, повторяя рассуждения, приведенные выше, видим, что $\widetilde{D^{\alpha} f_{i}}-$ аналитическая функция в $U\left(a_{\tau}, \rho\right)$ для всех $i \in \mathbb{N}_{k}$ и любых $\alpha \in \mathbb{N}^{n}$. Утверждение доказано.

Пусть $S=\left\{f_{1}, \ldots, f_{k}\right\} \subset \mathcal{F}_{a}$ - локальная дифференциальная система и пусть $W=U\left(a_{\tau}, \rho\right)-$ брус, в котором функции $\widetilde{D^{\alpha} f_{i}}$ аналитичны для всех $i \in \mathbb{N}_{k}$ и любых $\alpha \in \mathbb{N}^{n}$. Рассмотрим в пространстве $\mathbb{J}$ множество

$$
Z_{W}(S)=\left\{z \in W: \widetilde{D^{\alpha} f_{i}}(z)=0 \text { для всех } i \in \mathbb{N}_{k}, \alpha \in \mathbb{N}^{n}\right\}
$$

и точку $a \in W$. В дальнейшем $\mathbb{Z}_{a}(S)$ обозначает росток множества $Z_{W}(f)$ (см. монографию [20]).

УТВЕРЖДЕНИЕ 4.2. Если две локальные дифференциалъные системъ

$$
f=\left\{f_{1}, \ldots, f_{k}\right\} \subset \mathcal{F}_{a}, \quad g=\left\{g_{1}, \ldots, g_{s}\right\} \subset \mathcal{F}_{a}
$$

порождают один и тот же дифференциальный идеал в $\mathcal{F}_{a}$, то они задают одинаковые ростки, т.е.

$$
\mathbb{Z}_{a}(f)=\mathbb{Z}_{a}(g) .
$$

ДокАЗАТЕльСтво. Поскольку множества $f$ и $g$ порождают один и тот же дифференциальный идеал, любой ряд $g_{i} \in g$ представляется в виде

$$
g_{j}=\sum_{i, \alpha} c_{\alpha}^{i, j} D^{\alpha} f_{i}, \quad c_{\alpha}^{i, j} \in \mathcal{F}_{a} .
$$

Рассмотрим множества $Z_{W}(f)$ и $Z_{W}(g)$, принадлежащие брусу $U\left(a_{\tau}, \rho\right)$. Очевидно, из (4.3) следует включение

$$
\mathbb{Z}_{a}(g) \subseteq \mathbb{Z}_{a}(f)
$$


Используя приведенные выше рассуждения, получаем представление

$$
f_{i}=\sum_{j, \alpha} b_{\alpha}^{j, i} D^{\alpha} g_{j}, \quad b_{\alpha}^{j, i} \in \mathcal{F}_{a}
$$

и включение

$$
\mathbb{Z}_{a}(f) \subseteq \mathbb{Z}_{a}(g)
$$

Из формул (4.4) и (4.5) следует равенство (4.2).

Ниже приводятся понятия и результаты из работ [11], [12], которые понадобятся для доказательства основной теоремы.

ОПРЕДЕЛЕНИЕ. Орбитой подмножества $S$ в $\mathcal{F}_{a}$ называется множество

$$
O(S)=\left\{D^{\alpha} s: \alpha \in \mathbb{N}^{n}, s \in S\right\} .
$$

ОПРЕДЕЛЕНИЕ. Сходящийся дифференциальный ряд $f \in \mathcal{F}_{a}$ вида $f=u_{\alpha}^{i}+g$ называется разрешимым относительно $u_{\alpha}^{i}$, если $g$ не зависит от элементов орбиты $O\left(u_{\alpha}^{i}\right)$. Если ряд $f \in \mathcal{F}_{a}$ разрешим относительно $u_{\alpha}^{i}$, то символ st $f$ обозначает $u_{\alpha}^{i}$. Если $S$ - некоторое множество разрешимых рядов, то st $S=\{$ st $f: f \in S\}$.

ОПРЕДЕЛЕНИЕ. Пусть $S \subset \mathcal{F}_{a}$ - локальная дифференциальная система, состоящая из разрешимых сходящихся дифференциальных рядов. Предположим, что идеал $\langle\langle S\rangle\rangle \mathcal{F}_{a}$ обладает нормализованной системой образующих $\mathcal{B}$ и множество главных переменных $\mathcal{L}$ системы $\mathcal{B}$ удовлетворяет условию $\mathcal{L}=O($ st $S)$. Тогда $S$ называется пассивной системой идеала $\langle\langle S\rangle$.

Возникает задача о проверке локальной дифференциальной системы на пассивность. Для ее решения нужно ввести дополнительные понятия, частично использованные в работе [12].

Напомним, что предпорядком на множестве называется рефлексивное и транзитивное отношение, а строгим предпорядком - иррефлексивное и транзитивное отношение [21].

УтВЕРЖДЕНИЕ 4.3. Пусть $\left\{H_{\gamma}\right\}_{\gamma \in \Gamma}-$ разбиение множества $H$, где $Г$ - вполне упорядоченное множество. Тогда на $H$ можно задать предпорядок $\preceq$ строгий

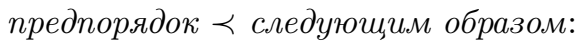

$$
\begin{aligned}
& h_{1} \preceq h_{2} \Longleftrightarrow \exists \gamma_{1}, \gamma_{2}: h_{1} \in H_{\gamma_{1}}, h_{2} \in H_{\gamma_{2}}, \gamma_{1} \leqslant \gamma_{2}, \\
& h_{1} \prec h_{2} \Longleftrightarrow \exists \gamma_{1}, \gamma_{2}: h_{1} \in H_{\gamma_{1}}, h_{2} \in H_{\gamma_{2}}, \gamma_{1}<\gamma_{2} .
\end{aligned}
$$

ДОкАЗАТЕЛЬСТво очевидно.

Пусть на множестве $M$ (слева) действует полугруппа $G$, т. е. задано отображение $(g, m) \rightarrow g m$ множества $G \times M$ в $M$, удовлетворяющее условию

$$
g_{1}\left(g_{2} m\right)=\left(g_{1} g_{2}\right) m, \quad m \in M, \quad g_{1}, g_{2} \in G .
$$

ОПреДЕЛЕНиЕ. Пусть на множестве $M$ действует полугруппа $G$ и разбиение $\left\{M_{\gamma}\right\}_{\gamma \in \Gamma}$ множества $M$ порождает строгий предпорядок $\prec$ согласно (4.7). Множество $M$ называется стратифицированным $G$-множеством, если для всех $g \in G$ выполняются условия:

1) если $m_{1} \prec m_{2}$, то $g m_{1} \prec g m_{2}$ для всех $m_{1}, m_{2} \in M$;

2) $m \prec g m$ для всех $m \in M$. 
Пусть $x^{0} \in \mathbb{K}^{n}, \mathbb{K}\left[\left\langle x_{1}, \ldots, x_{n}\right\rangle\right]_{x_{0}}$ - алгебра ростков аналитических функций в точке $x^{0}$, изоморфная соответствующей алгебре сходящихся степенных рядов. Рассмотрим точку $a \in \mathbb{J}$, стандартная проекция которой в $\mathbb{K}^{n}$ равна $x^{0}$, и, значит, декартовы координаты точки $x^{0}$ являются частью декартовых координат точки $a$. Обозначим через $\widehat{\mathcal{F}}_{a}$ множество $\mathcal{F}_{a} \backslash \mathbb{K}\left[\left\langle x_{1}, \ldots, x_{n}\right\rangle\right]_{x_{0}}$.

Любое разбиение $\left\{U_{\gamma}\right\}_{\gamma \in \Gamma}$ множества $U$ (см. соотношения (3.7)) порождает разбиение множества $\widehat{\mathcal{F}}_{a}$. Действительно, рассмотрим семейство множеств

$$
Y_{\gamma}=X \cup\left(\bigcup_{\Theta \leqslant \gamma^{\prime} \leqslant \gamma} U_{\gamma^{\prime}}\right)
$$

где $X$ определено в (3.7), $\Theta=\min _{\gamma \in \Gamma} \gamma$. Очевидно, справедливы формулы

$$
Y=\bigcup_{\gamma \in \Gamma} Y_{\gamma}, \quad Y_{\gamma^{\prime}} \subset Y_{\gamma^{\prime \prime}} \text { для всех } \gamma^{\prime}<\gamma^{\prime \prime} .
$$

Возьмем точку $a \in \mathbb{J}$ и рассмотрим цепь (по включению) подалгебр алгебры $\mathcal{F}_{a}$, заданных как

$$
\mathcal{F}_{a}^{\gamma}=\left\{f \in \mathcal{F}_{a}: \operatorname{iv}(f) \subset Y_{\gamma}\right\} .
$$

Здесь, как и раньше, iv $(f)$ означает множество переменных, от которых зависит ряд $f$. Тогда цепь $\left\{\mathcal{F}_{a}^{\gamma}\right\}_{\gamma \in \Gamma}$ порождает разбиение $\left\{\Phi_{a}^{\gamma}\right\}_{\gamma \in \Gamma}$ множества $\widehat{\mathcal{F}}_{a}$ на блоки

$$
\begin{aligned}
\Phi_{a}^{\gamma} & =\mathcal{F}_{a}^{\gamma} \backslash\left(\bigcup_{\gamma^{\prime}<\gamma} \mathcal{F}_{a}^{\gamma}\right), \quad \gamma>\Theta, \\
\Phi_{a}^{\Theta} & =\mathcal{F}_{a}^{\Theta} \backslash \mathbb{K}\left[\left\langle x_{1}, \ldots, x_{n}\right\rangle\right]_{x_{0}} .
\end{aligned}
$$

Кроме того, цепь подмножеств (4.8) множества $Y$ порождает цепь координатных подпространств

$$
J_{\gamma}=\left\{z \in \mathbb{J}: y(z)=0 \text { для всех } y \in Y \backslash Y_{\gamma}\right\}
$$

пространства Ј. Очевидно, верны формулы

$$
\bigcup_{\gamma \in \Gamma} J_{\gamma}=\mathbb{J}, \quad J_{\gamma^{\prime}} \subset J_{\gamma^{\prime \prime}} \text { для всех } \gamma^{\prime}<\gamma^{\prime \prime} .
$$

На множествах $U$ и $\widehat{\mathcal{F}}_{a}$ действие полугруппы $\mathbb{N}_{-0}^{n}=\mathbb{N}^{n} \backslash \overrightarrow{0}$, где $\overrightarrow{0}-$ кортеж из нулей, задается формулами

$$
\alpha u_{\beta}^{i}=u_{\alpha+\beta}^{i}, \quad \alpha f=D^{\alpha}(f)
$$

для любых $\alpha \in \mathbb{N}_{-0}^{n}$. Как следует из работы [11], $\widehat{\mathcal{F}}_{a}$ является стратифицированным $\mathbb{N}_{-0}^{n}$-множеством, если $U$ - стратифицированное $\mathbb{N}_{-0}^{n}$-множество.

В дальнейшем всюду предполагается, что $\widehat{\mathcal{F}}_{a}$ является стратифицированным $\mathbb{N}_{-0}^{n}$ множеством, наделенным соответствующим предпорядком (4.6) и строгим предпорядком (4.7). 
ОПРЕДЕЛЕНИЕ. Ряд $f \in \mathcal{F}_{a}$ вида

$$
f=u_{\alpha}^{i}+h, \quad h \prec u_{\alpha}^{i},
$$

называется упорядоченно разрешимым (относительно $u_{\alpha}^{i}$ ).

ОПРЕДЕЛЕНИЕ. Пусть $F-$ произвольный ряд из алгебры $\mathcal{F}_{a}$, а $f \in \mathcal{F}_{a}$ - упорядоченно разрешимый ряд относительно $u_{\alpha}^{i}$. Будем говорить, что ряд $F$ редуиируется $\kappa$ рлду $r \in \mathcal{F}_{a}$ относителъно $f$, если существует элемент $\delta \in \mathbb{N}^{n}$, для которого $u_{\alpha+\delta}^{i} \in \operatorname{iv}(F)$, и найдется ряд $q \in \mathcal{F}_{a}$ такой, что $F=q D^{\delta} f+r$, где $q \preceq F, r \preceq F$, $u_{\alpha+\delta}^{i} \notin \operatorname{iv}(r)$. Если ряд $F$ редуцируется к ряду $r$ относительно $f$, мы будем использовать обозначение $F \underset{f}{\rightarrow} r$.

В работе [12] доказано следующее утверждение.

УтВЕРЖДЕНИЕ 4.4. Пусть $F$ - произволъный ряд из алгебры $\mathcal{F}_{a}$ и $u_{\beta}^{i} \in \operatorname{iv}(F)$. Если ряд $f \in \mathcal{F}_{a}$ упорядоченно разрешим относительно $u_{\alpha}^{i}$ и существует $\delta \in \mathbb{N}^{n}$ maкое, что $u_{\beta}^{i}=u_{\alpha+\delta}^{i}$, mo $F \underset{f}{\rightarrow} r$.

ОПРЕДЕЛЕНИЕ. Подмножество $S$ в $\mathcal{F}_{a}$ называется слабо разрешимым, если каждый ряд $f \in S$ упорядоченно разрешим относительно некоторого $u_{\alpha}^{i}$. Элемент $u_{\alpha}^{i}$ называется старшим членом ряда $f$ и обозначается как lt $f$.

ОПРЕДЕЛЕНИЕ. Пусть $F \in \mathcal{F}_{a}$, а $S=\left\{f_{1}, \ldots, f_{k}\right\} \subset \mathcal{F}(a)$ - слабо разрешимое подмножество. Ряд $F$ редуцируется к ряду $r \in \mathcal{F}(a)$ относительно $S$, если существует конечная последовательность одношаговых редукций вида

$$
F \underset{f_{i_{1}}}{\longrightarrow} r_{1} \underset{f_{i_{2}}}{\longrightarrow} r_{2} \underset{f_{i_{3}}}{\longrightarrow} \cdots \underset{f_{i_{p}}}{\longrightarrow} r
$$

где $f_{i_{j}} \in S$. Эта последовательность редукций кратко обозначается как $F \underset{S}{\longrightarrow} r$.

ОПРЕДЕЛЕНИЕ. Ряд $f \in \mathcal{F}_{a}$ является нередуцируемым относительно слабо разрешимого множества $S$, если $\operatorname{iv}(f) \cap O($ lt $S)=\varnothing$, где lt $S=\{$ lt $f: f \in S\}$.

ОПРЕДЕЛЕНИЕ. Ряд $r \in \mathcal{F}_{a}$ называется нормалъной формой ряда $f \in \mathcal{F}_{a}$ относительно слабо разрешимого подмножества $S \subset \mathcal{F}_{a}$, если $f \underset{S}{\rightarrow} r$ и $r$ - ряд, нередуцируемый относительно $S$. Нормальная форма ряда $f$ относительно $S$ обозначается через $\mathrm{NF}(f \downarrow S)$.

В общем случае нормальная форма ряда относительно произвольного слабо разрешимого подмножества определена неоднозначно. Для однозначности нормальной формы достаточно, чтобы $S$ была пассивной системой [12].

Введем на $\mathbb{N}^{n}$ бинарную операцию $\diamond$ для $\alpha=\left(\alpha_{1}, \ldots, \alpha_{n}\right), \beta=\left(\beta_{1}, \ldots, \beta_{n}\right)$ положим

$$
\alpha \diamond \beta=\left(\mu_{1}, \ldots, \mu_{n}\right), \quad \mu_{i}=\max \left(\alpha_{i}, \beta_{i}\right)-\alpha_{i} .
$$

ОПРЕДЕЛЕНиЕ. Пусть $f_{1}, f_{2}$ - два разрешимых ряда из $\mathcal{F}_{a}$ вида

$$
f_{1}=u_{\alpha}^{i}+h_{1}, \quad f_{2}=u_{\beta}^{i}+h_{2},
$$


где $u_{\alpha}^{i}=$ lt $f_{1}, u_{\beta}^{i}=$ lt $f_{2}$. Тогда разность

$$
D^{\alpha \diamond \beta} f_{1}-D^{\beta \diamond \alpha} f_{2}
$$

называется $\tau$-рядом om $f_{1}, f_{2}$ и обозначается как $\tau\left(f_{1}, f_{2}\right)$.

ОПРЕДЕЛЕНИЕ. Пусть $S=\left\{f_{1}, \ldots, f_{k}\right\}$ - локальная дифференциальная система, являющаяся слабо разрешимым подмножеством в $\mathcal{F}_{a}$. Будем говорить, что система $S$ удовлетворяет условиям совместности, если для каждой пары $f_{i}, f_{j} \in S$ вида (4.14) соответствующий $\tau$-ряд (4.15) редуцируется к нулю относительно $S$.

Нижеследующее утверждение и теорема дают ответ на вопрос о принадлежности ряда дифференциальному идеалу.

УтВЕРЖДЕНИЕ 4.5. Пусть $S=\left\{f_{1}, \ldots, f_{k}\right\} \subset \mathcal{F}_{a}$ - пассивная система $u$ lt $S=$ st $S$. Ряд $f \in \mathcal{F}_{a}$ принадлежит дифференциалъному идеалу $I=\langle\langle S\rangle\rangle$ тогда и толъко тогда, когда $f$ редуиируется к нулю относительно $S$.

ДокАЗАТЕЛЬСтво. Если $f \underset{S}{\rightarrow} 0$, то из определения редукции следует, что

$$
f=\sum a_{\alpha}^{i} D^{\alpha} f_{i}, \quad f_{i} \in S
$$

т. e. $f \in I$.

Обратно, пусть $f \in I$. Как показано в работе [12], каждый ненулевой элемент из идеала $I$ зависит хотя бы от одного элемента из орбиты $O(\operatorname{lt} S)$. Если $f \underset{S}{\rightarrow} 0$, где ряд $r$ не редуцируется относительно $S$, то $r$ не зависит от элементов из $O($ lt $S)$. Таким образом, $r=0$.

Если система $S$ не является пассивной, то утверждение 4.5 в общем случае неверно, так как результат редукции определен неоднозначно.

Пример. Пусть дана система $S=\left\{f_{1}=u_{1,1}+u, f_{2}=u_{0,2}-u\right\}$ и ряд $f=u_{1,2}-u_{1,0}$. Данная система не пассивна, поскольку $D_{2} f_{1}-D_{1} f_{2}=u_{1,0}+u_{0,1}$ не зависит от элементов орбиты $O\left(\operatorname{lt}\left(u_{0,2}\right), \operatorname{lt}\left(u_{1,2}\right)\right)$. Ряд $f$ можно представить двумя способами: $f=D_{2} f_{1}-u_{1,0}-u_{0,1}$ и $f=D_{1} f_{2}$, т. е. в первом случае $f$ редуцируется к $-u_{1,0}-u_{0,1}$, а во втором - к нулю.

Заметим, что если $f \in \mathcal{F}_{b}$, то $f \in \mathcal{F}_{a}$ для бесконечного числа точек $a$. Пусть $S=\left\{f_{1}, \ldots, f_{k}\right\} \subset \mathcal{F}_{b}$ - локальная дифференциальная система и $f_{i}(b)=0$ для всех $i \in \mathbb{N}_{k}$. Будем называть точки $a, b \in \mathbb{J}$ эквивалентными по модулю $S$ (и писать $a \sim b \bmod S)$, если $f_{i} \in \mathcal{F}_{a}$ и $f_{i}(a)=0$ для всех $f_{i} \in S$.

Теорема 4.1. Пусть $S=\left\{f_{1}, \ldots, f_{k}\right\} \subset \mathcal{F}_{b}-$ локальная дифференциальная система, являющаяся слабо разрешимым подмножеством в $\mathcal{F}_{b}$, удовлетворяющая условиям совместности, $u f_{i}(b)=0$ для всех $f_{i} \in S$. Тогда $S$ - пассивная система, существует точка $a \in \mathbb{J}$, эквивалентнал $b$ по модулю $S, u$ брус $W$, содержащий эту точку, такие, что множество $Z_{W}(f)$ вида (4.1) является аналитическим многообразием в $W$ с системой параметров $Y \backslash$ lt $S$.

ДокАзАТЕЛьство. Пассивность системы $S$ доказана в работе [11]. Там же показано, что существует точка $a \in \mathbb{J}($ где $a \sim b \bmod S)$ такая, что $f(a)=0$ для всех 
рядов $f$ из орбиты $O(S)$. Кроме того, доказано, что существует нормализованная система $\mathcal{B}$ образующих идеала $\langle\langle S\rangle\rangle$, множество главных переменных которой совпадает с lt $S$.

Не ограничивая общности, можно считать, что $S$ - нормализованное множество (или, в терминологии монографии [22], ортономное), так как согласно результатам работ [11], [12] $S$ - пассивная система и существует каноническое множество $S^{\prime}$ такое, что $\left\langle\langle S\rangle=\left\langle\left\langle S^{\prime}\right\rangle\right\rangle\right.$. Тогда из утверждения 4.2 следует, что ростки $\mathbb{Z}_{a}(S)$ и $\mathbb{Z}_{a}\left(S^{\prime}\right)$ совпадают. Итак, достаточно доказать, что найдется брус $W$, содержащий точку $a$, такой, что аналитическое множество $Z_{W}\left(S^{\prime}\right)$ есть многообразие в брусе $W$.

Согласно утверждению 2.2 нужно показать, что множество аналитических функций из $\widetilde{\mathcal{B}}$, соответствующее нормализованной системе образующих $\mathcal{B}$ идеала $\left\langle\left\langle S^{\prime}\right\rangle\right.$, задано в некотором брусе $V \subset \mathbb{J}$. Как следует из утверждения 3.1, существует брус $U\left(a_{\tau}, \rho\right)$, в котором все функции из $\overparen{O\left(S^{\prime}\right)}$ являются аналитическими. Поэтому достаточно установить, что множество аналитических функций из $\widetilde{\mathcal{B}}$ также определено в $U\left(a_{\tau}, \rho\right)$. Для этого вспомним, как в работе [11] строилась нормализованная система образующих.

Как отмечалось ранее, разбиение множества $U$ из (3.7) порождает цепь (4.9) подалгебр $\mathcal{F}_{a}^{\gamma}$, цепь (4.11) подпространств $J_{\gamma}$ и разбиение (4.10) $\left\{\Phi_{a}^{\gamma}\right\}_{\gamma \in \Gamma}$. Обозначим через $a_{\gamma}$ естественную проекцию точки $a$ на подпространство $J_{\gamma}$. Введем также следующие обозначения:

$$
\begin{gathered}
\gamma_{0}=\min \left\{\gamma \in \Gamma: O(S) \cap \mathcal{F}_{a}^{\gamma} \neq \varnothing\right\}, \quad \Pi_{\gamma}=U\left(a_{\tau}, \rho\right) \cap J_{\gamma}, \\
O_{\gamma}=O(S) \cap \mathcal{F}_{a}^{\gamma}, \quad C_{\gamma}=O(S) \cap \Phi_{a}^{\gamma},
\end{gathered}
$$

где $\mathcal{F}_{a}^{\gamma}$ и $\Phi_{a}^{\gamma}$ задаются формулами (4.9) и (4.10) соответственно. Очевидно, для любого $\gamma_{\star} \geqslant \gamma$ верна формула

$$
O_{\gamma_{\star}}=C_{\gamma_{\star}} \cup\left(\bigcup_{\gamma_{0} \leqslant \gamma<\gamma_{\star}} C_{\gamma}\right)
$$

Обозначим через $\left\langle O_{\gamma}\right\rangle_{a_{\gamma}}$ идеал алгебры $\mathcal{F}_{a}^{\gamma}$, порожденный множеством $O_{\gamma}$.

Используя принцип трасфинитной индукции, покажем, что:

1) для любого $\gamma \geqslant \gamma_{0}$ существует нормализованная система образующих $B_{\gamma}$ идеала $\left\langle O_{\gamma}\right\rangle_{a_{\gamma}}$ алгебры $\mathcal{F}_{a}^{\gamma}$ такая, что $L_{\gamma}=$ lt $O_{\gamma}$, где $L_{\gamma}-$ множество главных переменных системы $B_{\gamma}$;

2 ) все функции из $\widetilde{B}_{\gamma}$ являются аналитическими в $\Pi_{\gamma}$.

При $\gamma=\gamma_{0}$ верны равенства

$$
O_{\gamma_{0}}=S \cap \mathcal{F}_{a}^{\gamma_{0}}=S \cap \Phi_{a}^{\gamma_{0}}=C_{\gamma_{0}}
$$

Несложно видеть (см. также работу [11]), что множество $O_{\gamma_{0}}$ является нормализованной системой образующих идеала $\left\langle O_{\gamma_{0}}\right\rangle_{\gamma_{0}}$. Поэтому согласно утверждению 2.2 аналитическое множество

$$
Z_{\gamma_{0}}=\left\{z \in \Pi_{\gamma_{0}}: \tilde{f}(z)=0 \text { для всех } f \in \tilde{O}_{\gamma_{0}}\right\}
$$

является многообразием в $\Pi_{\gamma_{0}}$. Очевидно, $L_{\gamma_{0}}=\operatorname{lt} O_{\gamma_{0}}$. 
Теперь предположим, что для всех значений $\gamma$, удовлетворяющих неравенствам $\gamma_{0} \leqslant \gamma<\gamma_{\star}$, верно следующее утверждение: существует нормализованная система образующих $B_{\gamma}$ идеала $\left\langle O_{\gamma}\right\rangle_{a_{\gamma}}$ такая, что $L_{\gamma}=\operatorname{lt} O_{\gamma}$ и все функции из $\widetilde{B}_{\gamma}$ являются аналитическими в $\Pi_{\gamma}$. Покажем, что эти свойства имеют место и при $\gamma=\gamma_{\star}$.

Согласно равенству (4.16) и предположению индукции множество

$$
G_{\gamma_{\star}}=C_{\gamma_{\star}} \cup\left(\bigcup_{\gamma_{0} \leqslant \gamma<\gamma_{\star}} B_{\gamma}\right)
$$

является системой образующих идеала $\left\langle O_{\gamma_{\star}}\right\rangle_{a_{\gamma_{\star}}}$. В работе [11] с использованием множества $G_{\gamma_{\star}}$ построена нормализованная система образующих $B_{\gamma_{\star}}$. В рассматриваемом нами случае, когда $S^{\prime}$ - каноническое множество, имеется своя специфика. Именно, любой ряд $f \in C_{\gamma_{\star}}$ имеет вид $f=u_{\alpha}^{i}+g$, где $g \prec u_{\alpha}^{i}$, при этом в силу формулы дифференцирования (3.8) ряд $g \in \mathcal{F}_{a}^{\gamma}$ для $\gamma<\gamma_{\star}$ является полиномом от главных переменных нормализованной системы образующих $B_{\gamma}$. Согласно соотношению (2.5) коэффициенты этого полинома являются степенными рядами, сходящимися в $\Pi_{\gamma}$, и зависят только от параметрических переменных системы $B_{\gamma}$. Если выразить главные переменные, входящие в $g$, через ряды из $B_{\gamma}$, то получим ряд $f_{\star} \in B_{\gamma_{\star}}$, сходящийся в $\Pi_{\gamma_{\star}}$.

Как следует из предыдущих рассуждений, множество $B=\bigcup_{\gamma_{0} \leqslant \gamma} B_{\gamma}$ является нормализованной системой образующих идеала $\langle\langle S\rangle\rangle$ и соответствующее множество $\widetilde{B}$ состоит из функций, аналитических в брусе $U\left(a_{\tau}, \rho\right)$. Для завершения доказательства теоремы остается сослаться на утверждение 2.2.

ЗАмечАниЕ. Стандартным образом можно определить касательные расслоения и векторные поля на многообразиях, а также пучки ростков аналитических функций [14], [23].

ПримеР. Рассмотрим нелинейное уравнение Дюбрей-Жакотен [24]

$$
\Delta \psi+\frac{\rho^{\prime}}{2 \rho}\left[(\nabla \psi)^{2}+y\right]+F=0,
$$

где $\psi$ - функция тока, зависящая от $x$ и $y, \rho$ - плотность жидкости, зависящая от $\psi$, $\Delta$ - оператор Лапласа, $(\nabla \psi)^{2}=\psi_{x}^{2}+\psi_{y}^{2}, F-$ некоторая функция от $\psi$. Данное уравнение описывает плоское стационарное течение стратифицированной жидкости в поле силы тяжести. В наших обозначениях (декартовых координатах на $\mathbb{J}$ ) данное уравнение задается рядом

$$
u_{(2,0)}+u_{(0,2)}+\frac{\rho^{\prime}}{2 \rho}\left[u_{(1,0)}^{2}+u_{(0,1)}^{2}+x_{2}\right]+F
$$

где $\rho$ и $F$ - аналитические функции от $u_{(0,0)}$. В дальнейшем будем использовать привычные обозначения и писать, например, $u_{x}, u_{y}$ вместо $u_{(1,0)}, u_{(0,1)}$.

Найдем, при каких функциях $\rho$ и $F$ уравнение (4.17) допускает обобщенное разделение переменных, т. е. решения вида $\psi=G(\alpha(x)+\beta(y))$, где $\alpha$ и $\beta$ - функции от $x$ и $y$ соответственно, $G$ - некоторая функция. Такого типа решения нелинейного уравнения Лапласа описаны в монографии [20]. 
Задачу можно переформулировать как задачу исследования совместности системы, образованной уравнением (4.17) и уравнением

$$
\phi(\psi)_{x y}=0,
$$

где $\phi-$ функция, обратная к $G$.

Введем новую функцию $w=\phi(\psi)$, тогда уравнения $(4.17),(4.18)$ примут вид

$$
\begin{aligned}
\Delta w+(\nabla w)^{2} r+y f+g & =0, \\
w_{x y} & =0 .
\end{aligned}
$$

Здесь $r, f, g$ - некоторые функции от $w$. Кратко опишем исследование совместности системы (4.19), (4.20), используя предложенные выше методы. Операторы полного дифференцирования (3.8) будем обозначать через $D_{x}, D_{y}$. Вычисления объемные, поэтому мы применили систему компьютерной алгебры Maple [10].

Действуя оператором $D_{x} D_{y}$ на уравнение (4.19) и редуцируя полученное выражение относительно $(4.20)$, т. е. заменяя все производные $w_{x y}, w_{x x y}, w_{x x x y}$ на 0 , получаем уравнение второго порядка. Редуцируя последнее уравнение относительно $(4.19)$, получаем некоторый полином $E_{1}$ по $w_{x}, w_{y}$. Несложно показать, что если все коэффициенты этого полинома обращаются в ноль, то это возможно только тогда, когда уравнение (4.19) имеет вид

$$
\Delta w+(\nabla w)^{2}+a y+b w=0, \quad a, b \in \mathbb{R} .
$$

Обобщенный метод разделения переменных для этого уравнения можно найти в книге [20], там же представлены соответствующие картины линий тока. Заметим, что дифференциальный идеал, порожденный последним уравнением и уравнением (4.19), обладает нормализованной системой образующих. Главными переменными этой системы образующих можно считать $D_{x}^{n} D_{y}^{m} w_{x x}, D_{x}^{n} D_{y}^{m} w_{x y}$ при $n, m \in \mathbb{N}$.

Если полином $E_{1}$ ненулевой, то, действуя на него оператором $D_{x}$ и редуцируя полученное выражение относительно (4.19), (4.20), мы получаем некоторое соотношение. Если же подействовать на полином $E_{1}$ оператором $D_{y}$ и редуцировать полученное выражение относительно (4.19), (4.20), то получим некоторое новое соотношение. Используя уравнения (4.19), (4.20), из этих двух соотношений можно исключить все переменные $w_{x y}, w_{x x}, w_{y y}$ и получить новый полином $E_{2}$ от $w_{x}, w_{y}$.

Предположим теперь, что полиномы $E_{1}$ и $E_{2}$ отличаются лишь множителем. Это дает семь обыкновенных дифференциальных уравнений для трех функций $f, g, r$, стоящих в левой части уравнения (4.19). Из этих семи уравнений можно получить три дифференциальных уравнения первого порядка, решая которые, находим

$$
r=\frac{1}{-2 w+c_{0}}, \quad g=\frac{c_{1}}{-2 w+c_{0}}, \quad f=\frac{c_{2}}{-2 w+c_{0}},
$$

где $c_{0}, c_{1}, c_{2} \in \mathbb{R}$. Следовательно, уравнение (4.19) можно записать как

$$
\Delta w+(\nabla w)^{2} \frac{1}{-2 w+c_{0}}+y \frac{c_{2}}{-2 w+c_{0}}+\frac{c_{1}}{-2 w+c_{0}}=0 .
$$

Несложно видеть, что общим решением последнего уравнения и уравнения (4.20) является многочлен

$$
w=b_{1} y+a_{2} x^{2}+a_{1} x+a_{0},
$$


где $b_{1}, a_{1} \in \mathbb{R}, a_{2}=c_{2} / 4 b_{1}, a_{0}=\left(c_{1}+2 c_{0} a_{2}+a_{1}^{2}+b_{1}^{2}\right) / 4 a_{2}$. Если ввести новые переменные

$$
y^{\prime}=y+\frac{c_{1}}{c_{2}}, \quad w^{\prime}=w-\frac{c_{0}}{2},
$$

то уравнение (4.21) примет вид

$$
\Delta w^{\prime}+\frac{\left(\nabla w^{\prime}\right)^{2}}{-2 w^{\prime}}+\frac{c_{2} y^{\prime}}{-2 w^{\prime}}=0
$$

В результате замены $w^{\prime}=\psi^{2}-c_{2} / 4$ получаем уравнение для функции тока в форме Дюбрей-Жакотен:

$$
\Delta \psi+\frac{c_{2}}{c_{2} \psi-4 \psi^{3}}\left[(\nabla \psi)^{2}+y\right]=0
$$

Найденное выше решение этого уравнения имеет вид $\psi=\left(w-c_{0} / 2+c_{2} / 4\right)^{1 / 2}$, где функция $w$ задана формулой (4.22). Некоторые другие уравнения Дюбрей-Жакотен, допускающие обобщенное разделение переменных, приведены в работе [25].

В книге [19] представлена групповая классификация уравнения Дюбрей-Жакотен. Из результатов этой классификации следует, что уравнение (4.23) допускает двумерную алгебру симметрий, и найденное в настоящей работе решение (4.22) не является инвариантным.

\section{5. ЗАКЛЮЧЕНИЕ}

В представленной работе мы рассмотрели алгебру $\mathcal{F}_{a}$ сходящихся степенных рядов, вместо нее можно изучать кольцо ростков гладких (бесконечно дифференцируемых) вещественнозначных функций, при этом большинство наших результатов остается справедливым. Наши рассуждения, в частности, основаны на теореме деления Вейерштрасса, но утверждение о единственности остатка имеет место и в гладком случае, если у соответствующего идеала существует нормализованная система образующих. Это связано с тем, что в данном случае теорема Вейерштрасса вытекает из теоремы о неявной функции [26].

Несомненный интерес представляет распространение описанного подхода на разностные алгебры и уравнения. K сожалению, при изучении разностных уравнений нельзя ограничиться локальными алгебрами. Тем не менее симметрии разностных уравнений были введены Дородницыным [27] и активно изучаются в настоящее время.

\section{Список литературы}

[1] Д. Кокс, Дж. Литтл, Д. О’Ши, Идеаль, многообразия и алгоритмъь, Мир, М., 2000.

[2] Б. Бухбергер, "Базисы Гребнера. Алгоритмический метод в теории полиномиальных идеалов", Компьютерная алгебра. Символические и алгебрачческие вычисления, ред. Б. Бухбергер, Дж. Коллинз, Р. Лоос, Мир, М., 1986, 331-372.

[3] Ch. Riquier, Les systèmes d'équations aux dérivées partielles, Gauthier-Villars, Paris, 1909.

[4] M. Janet, Leçons sur les systémes des équations aux dérivées partielles, Gauthier-Villars, Paris, 1929.

[5] Ж. Поммаре, Системь уравнений с частными производными и псевдогруппы Ли, Мир, М., 1983.

[6] А. М. Виноградов, И. С. Красильщик, В. В. Лычагин, Введение в геометрию нелинейных дифференииалъных уравнений, Наука, М., 1986. 
[7] V.V. Zharinov, Lecture Notes on Geometrical Aspects of Partial Differential Equations, Series on Soviet and East European Mathematics, 9, World Sci., Singapore, 1992.

[8] W. M. Seiler, Involution: The Formal Theory of Differential Equations and its Applications in Computer Algebra, Algorithms and Computation in Mathematics, 24, Springer, New York, 2010.

[9] M. Marvan, Found. Comput. Math., 9:6 (2009), 651-674.

[10] Maple, http://www.maplesoft.com/products/Maple/index.aspx.

[11] О. В. Капцов, Программирование, 40:2 (2014), 32-40.

[12] О. В. Капцов, ТМФ, 183:3 (2015), 342-358.

[13] Г. Грауэрт, Р. Реммерт, Аналитические локальные алгебры, Наука, М., 1988.

[14] Р. Ганнинг, Х. Росси, Аналитические функиии многих комплексных переменных, Мир, M., 1969.

[15] Ж.-П. Серр, Алгебры Ли и группы Ли, Мир, М., 1969.

[16] Н. Бурбаки, Алгебра. Многочлены и поля. Упорядоченные группы, Наука, М., 1965.

[17] Л. В. Овсянников, Групповой анализ дифференииалъных уравнений, Наука, М., 1978.

[18] А. М. Виноградов, И. С. Красильщик (ред.), Симметрии и законы сохранения уравнений математической физики, Факториал, М., 1997.

[19] V.K. Andreev, O.V. Kaptsov, V.V. Pukhnachov, A.A. Rodionov, Applications of Group-Theoretical Methods in Hydrodynamics, Springer, Amsterdam, 2010.

[20] Н. Бурбаки, Общая топология. Основные структуры, Наука, М., 1968.

[21] А. И. Белоусов, С. Б. Ткачев, Дискретная математика, Изд-во МГТУ, М., 2004.

[22] J. F. Ritt, Differential Algebra, Dover, New York, 1966.

[23] Д. Мамфорд, Красная книга о многообразиях и схемах, МЦМНО, М., 2007.

[24] C.-S. Yih, Stratified Flows, Academic Press, New York, 1980.

[25] Ю.В. Шанько, Выч. технологии, 6:5 (2001), 106-117.

[26] М. Голубицкий, В. Гийемин, Устойчивые отображения и их особенности, Мир, М., 1977.

[27] В. А. Дородницын, "Группы преобразований в сеточных пространствах", Итоги науки и техн. Сер. Соврем. пробл. матем. Нов. достиж., 34, ВИНИТИ, М., 1989, 149-191.

Поступила в редакцию 26.10 .2015 ,

после доработки 2.12.2015 